To appear on the Proceedings of the 13th ICATPP Conference on Astroparticle, Particle, Space Physics and Detectors for Physics Applications,

Villa Olmo (Como, Italy), 3-7 October, 2011, to be published by World Scientific (Singapore).

\title{
ELECTRICAL CHARACTERIZATION OF SiPM AS A FUNCTION OF TEST FREQUENCY AND TEMPERATURE
}

\author{
M.J. Boschini ${ }^{1,3}$, C. Consolandi ${ }^{1}$, P.G. Fallica ${ }^{4}$, M. Gervasi ${ }^{1,2}$, D. Grandi ${ }^{1}$, \\ M. Mazzillo ${ }^{4}$, S. Pensotti ${ }^{1,2}$, P.G. Rancoita ${ }^{1}$, D. Sanfilippo ${ }^{4}$, \\ M. Tacconi ${ }^{1 *}$ and G. Valvo ${ }^{4}$ \\ ${ }^{1}$ Istituto Nazionale di Fisica Nucleare, INFN Milano-Bicocca, Milano (Italy) \\ ${ }^{2}$ Department of Physics, University of Milano Bicocca, Milano (Italy) \\ ${ }^{3}$ CILEA, Segrate (MI) (Italy) \\ ${ }^{4}$ STMicroelectronics, Catania (Italy) \\ E-mail: mauro.tacconi@mib.infn.it
}

\begin{abstract}
Silicon Photomultipliers (SiPM) represent a promising alternative to classical photomultipliers for the detection of photons in high energy physics and medical physics, for instance. In the present work, electrical characterizations of test devices - manufactured by STMicroelectronics - are presented. SiPMs with an area of $3.5 \times 3.5 \mathrm{~mm}^{2}$ and a cell pitch of $54 \mu \mathrm{m}$ were manufactured as arrays of $64 \times 64$ cells and exhibiting a fill factor of $31 \%$. The capacitance of SiPMs was measured as a function of reverse bias voltage at frequencies ranging from about $20 \mathrm{~Hz}$ up to $1 \mathrm{MHz}$ and temperatures from $310 \mathrm{~K}$ down to $100 \mathrm{~K}$. Leakage currents were measured at temperatures from $410 \mathrm{~K}$ down to $100 \mathrm{~K}$. Thus, the threshold voltage - i.e., the voltage above a SiPM begins to operate in Geiger mode - could be determined as a function of temperature. Finally, an electrical model capable of reproducing the frequency dependence of the device admittance is presented.
\end{abstract}

\section{Introduction}

In recent years Silicon Photomultipliers (SiPM) has been developed for usage in photon detection. The high gain, insensitivity to magnetic field and low reverse bias voltage of operation make SiPM a promising candidates as replacement of classical photomultipliers ${ }^{1}$ in several of their applications. The SiPM is an array of parallel-connected single photon avalanche diodes $(\mathrm{SPAD})^{2}$. Every SPAD operates in Geiger mode with a quenching resistor in series to prevent an avalanche multiplication process from taking place. The overall output of a SiPM depends on how many SPADs are 
simultaneously ignited ${ }^{3,4}$.

The current SiPM device was manufactured by STMicroelectronics (details of the technology can be found in Refs. ${ }^{2,5}$ ). It consisted of an array of $64 \times 64$ SPAD cells with $3.5 \times 3.5 \mathrm{~mm}^{2}$ effective area, a cell pitch of $54 \mu \mathrm{m}$ and a fill factor of $\approx 31 \%$.

In the present article, the electrical characteristics of these devices are shown as function of test frequency from $1 \mathrm{MHz}$ down to about $20 \mathrm{~Hz}$ and temperature from $410 \mathrm{~K}$ down to $100 \mathrm{~K}$ (Sects. 2-2.2). In addition, in Sects. 3 and 3.1 an electrical model is discussed and compared with data obtained from dependencies of capacitance and resistance on the test frequency for both a photodiode and a SiPM devices.

\section{Electrical Characteristics of SiPM Devices}

The electrical characteristics of SiPM devices were investigated as function of applied reverse bias voltage $\left(V_{\mathrm{r}}\right)$ and temperature. The capacitance response was also studied as a function of test frequency of the capacimeter employed for such a measurement (Sect. 2.1).

Furthermore (Sect. 2.2), the measurements of the SiPM leakage current allowed one to determine (as a function of temperature) the value (and its temperature dependence) of the so-called threshold voltage $\left(V_{\mathrm{th}}\right)$, i.e., the

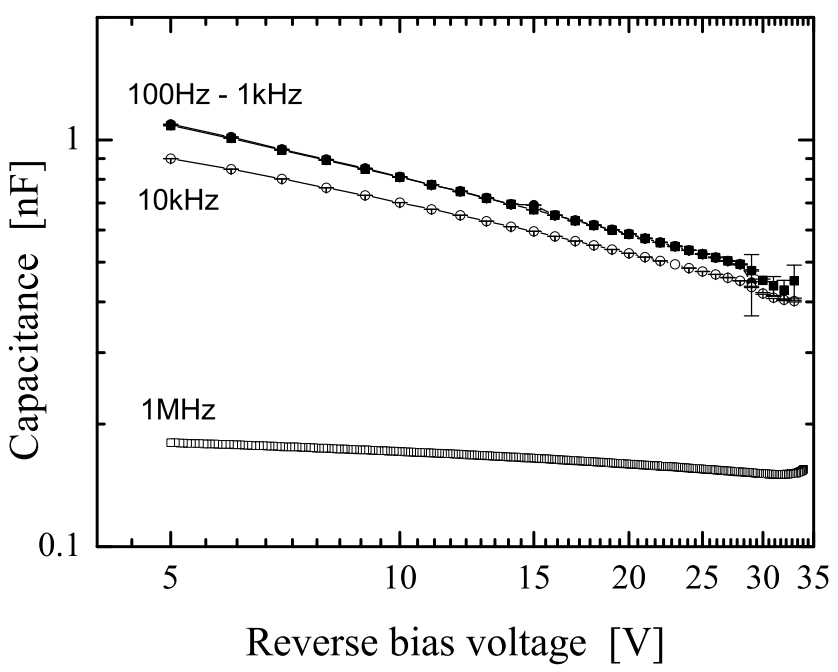

Fig. 1. Capacitance (in $\mathrm{nF}$ ) as a function of reversed bias voltage (in $\mathrm{V}$ ) using the LCZ meter with test frequencies of $100 \mathrm{~Hz}(\bullet), 1 \mathrm{kHz}(\boldsymbol{\square}), 10 \mathrm{kHz}(\circ)$ and $\mathrm{BC}$ with $1 \mathrm{MHz}(\square)$. 


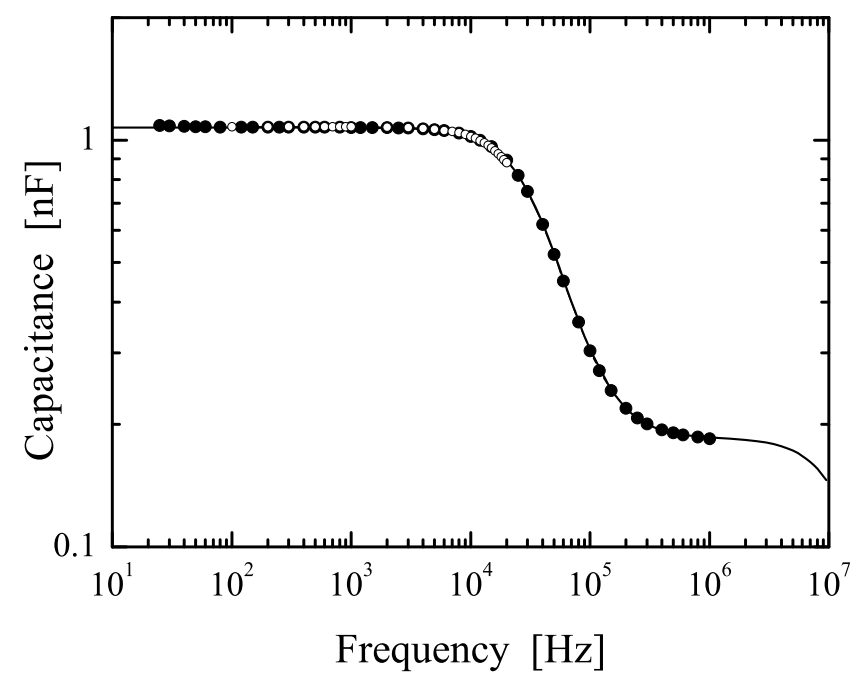

Fig. 2. Capacitance (in $\mathrm{nF}$ ) as a function of the frequency (in $\mathrm{Hz}$ ) for a sample operated with a reverse bias of $5 \mathrm{~V}$ : data points indicated with $\bullet(0)$ were obtained using the LCR (LCZ) meter, the continuous line is obtained from Eq. (9) (see discussion in Sect. 3.1).

reverse bias voltage above which a SiPM begins to operate in Geiger mode.

\subsection{Capacitance Response}

The capacitance response of SiPM devices was systematically measured as a function of reverse bias voltage applied and test frequency at $300 \mathrm{~K}$ (i.e., at room temperature). Moreover, using a liquid nitrogen cryostat, at a few fixed frequencies the capacitance was also measured as a function of reverse bias voltage and temperature from $310 \mathrm{~K}$ down to $100 \mathrm{~K}$. For these purposes three instruments were employed, i.e., a Boonton capacimeter (BC) (using its internally provided power source and a test frequency of $1 \mathrm{MHz}$ ), an LCZ (Agilent Technologies 4276A) and LCR (Agilent Technologies 4284A) meters with the reverse bias supplied to the device from an external power supply. The LCZ meter employed test frequencies from $100 \mathrm{~Hz}$ up to $20 \mathrm{KHz}$; the LCR meter from $\approx 20 \mathrm{~Hz}$ up to $1 \mathrm{MHz}$. Furthermore, the measurements were performed selecting the parallel equivalent-circuit mode of the LCR and LCZ meters for the device under test (DUT). Thus, the device response is assumed to be that one from a parallel capacitor-resistor circuit (see discussion in Sect. 3). Finally, the currently reported experimental errors 

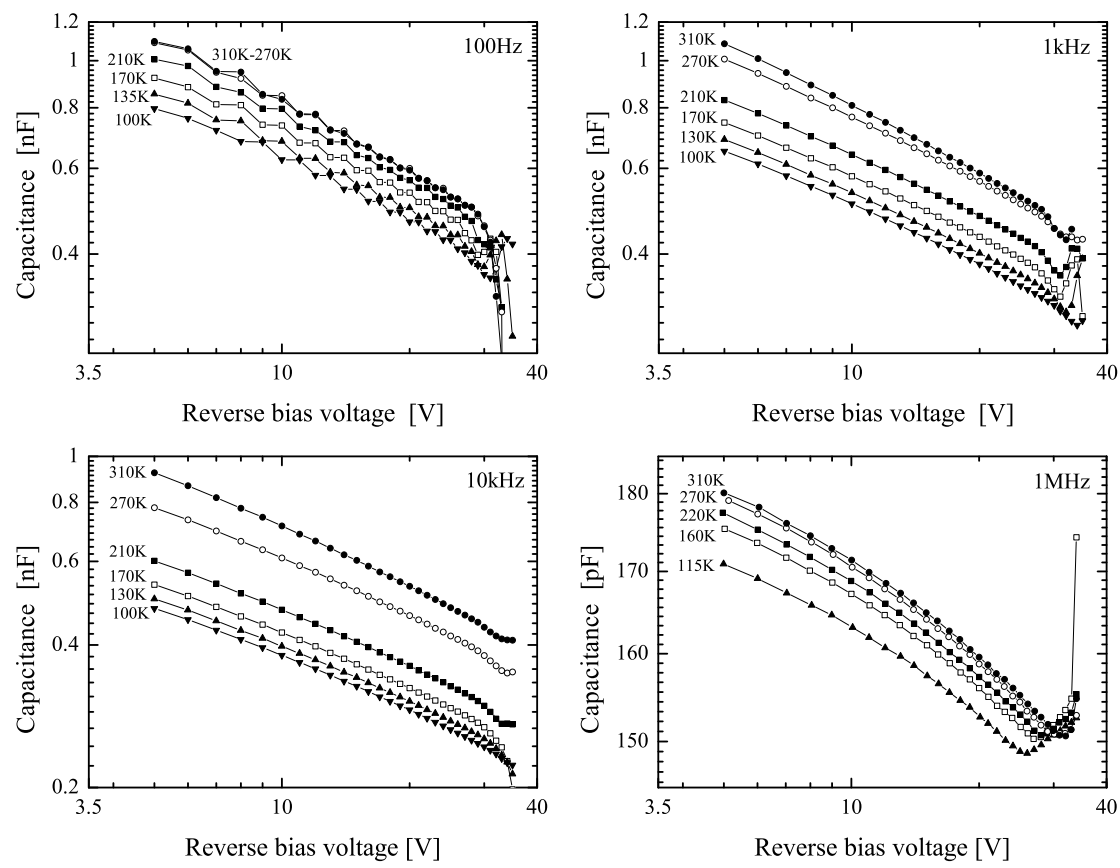

Fig. 3. Capacitance response (in $\mathrm{nF}$ or $\mathrm{pF}$ ) obtained using the $\mathrm{LCZ}$ meter as a function of reverse bias applied (in V) and temperature from $310 \mathrm{~K}$ down to $100 \mathrm{~K}: 100 \mathrm{~Hz}$ (upper left), $1 \mathrm{kHz}$ (upper right), $10 \mathrm{kHz}$ (bottom left) and - using the $\mathrm{BC}-1 \mathrm{MHz}$ (bottom right).

are the standard deviations obtained using 50 subsequent measurements of the device capacitance under the same experimental conditions.
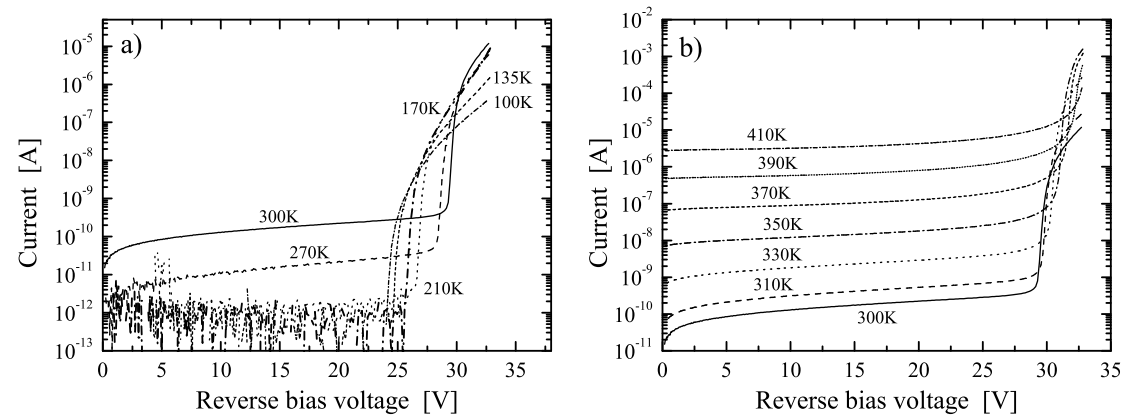

Fig. 4. (a) Leakage current (in A) as a function of reverse bias voltage (in V) from 310 down to $100 \mathrm{~K}$; (b) leakage current (in A) as a function of reverse bias voltage (in V) from 300 up to $410 \mathrm{~K}$. 
In Fig. 1, the capacitance response of a SiPM using frequencies of $100 \mathrm{~Hz}$, $1 \mathrm{kHz}, 10 \mathrm{kHz}$ and $1 \mathrm{MHz}$ is shown as a function of $V_{\mathrm{r}}$ at $300 \mathrm{~K}$. Above $1 \mathrm{kHz}$, the measured capacitance decreases with the increase of the test frequency. In Fig. 2, the capacitance response - determined using both LCZ and LCR meters - is shown for a device operated with a reverse bias voltage of $5 \mathrm{~V}$ at $300 \mathrm{~K}$. It can be observed that above $\approx 10 \mathrm{kHz}$ the measured capacitance largely decreases with the increase of the test frequency of the instrument up to achieving an almost constant response above a few hundreds of $\mathrm{kHz}$.

In Fig. 3, the capacitance response using $100 \mathrm{~Hz}, 1 \mathrm{kHz}, 10 \mathrm{kHz}$ and $1 \mathrm{MHz}$ is shown as a function of $V_{\mathrm{r}}$ (in $\mathrm{V}$ ) and temperature from $310 \mathrm{~K}$ down to $100 \mathrm{~K}$. The measured capacitance is observed to decrease with decreasing temperature. This behavior is also observed for frequencies lower than $\approx 10 \mathrm{kHz}$; while a similar capacitance response was exhibited at $300 \mathrm{~K}$ (see Fig. 1).

\subsection{Current-Voltage Characteristics}

The dependence of leakage current on reverse bias was studied as a function of temperature from $410 \mathrm{~K}$ down to $100 \mathrm{~K}$ as shown in Fig. 4. With increasing $V_{\mathrm{r}}$ the leakage current does not vary by more than an order of magnitude until the multiplication regime is ignited, i.e., so far the threshold voltage, $V_{\mathrm{th}}$, is reached. $V_{\mathrm{th}}$ is the voltage corresponding to the value of leakage current at the intercept between the two $I$ versus $V$ curves obtained, the first when the multiplication regime is sharply starting and the second one at lower voltages, i.e., before the multiplication occurs: an example regarding the determination of the value of $V_{\text {th }}$ at $270 \mathrm{~K}$ is reported in Fig. 5(a). As
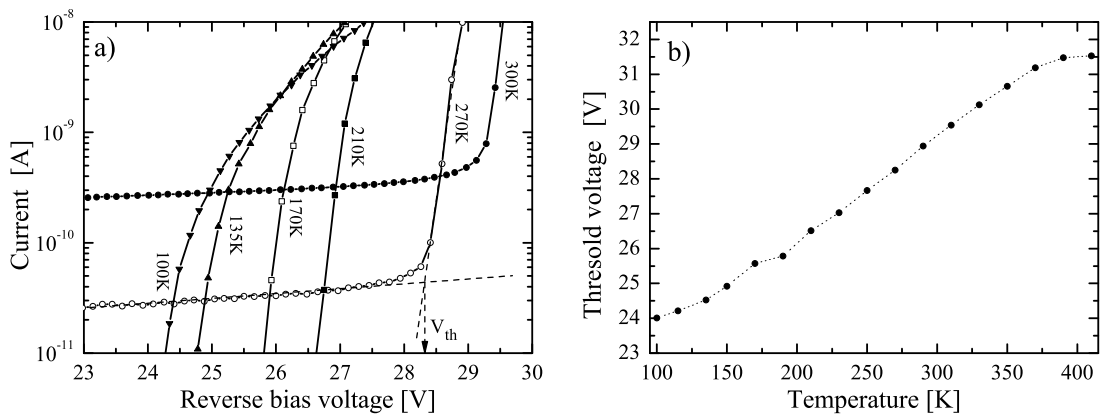

Fig. 5. (a) Leakage current (in A) as a function of reverse bias voltage applied (in V) and an example of threshold voltage, $V_{\text {th }}$ in $\mathrm{V}$, determination at $270 \mathrm{~K}$; (b) threshold voltage (in $\mathrm{V}$ ) as a function of temperature (in $\mathrm{K}$ ). 

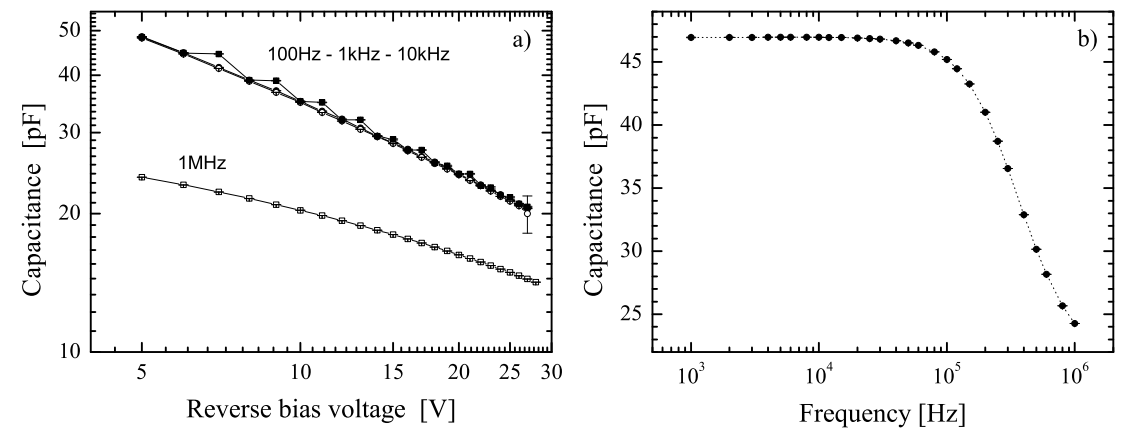

Fig. 6. (a) Capacitance (in $\mathrm{pF}$ ) of a photodiode as a function of reverse bias voltage (in V) at $100 \mathrm{~Hz}, 1 \mathrm{kHz}, 10 \mathrm{kHz}$ (using the LCZ meter) and $1 \mathrm{MHz}$ (using the $\mathrm{BC}$ ); (b) capacitance (in $\mathrm{pF}$ ) of a photodiode as a function of test frequency in $\mathrm{Hz}$ (using the LCR meter) with an applied reverse biased voltage of $6 \mathrm{~V}$.

shown in Fig. 5(b), the threshold voltage lowers - thus, SPADs turn into a Geiger-mode regime at progressively lower reverse bias voltages - with lowering temperature at the rate of $\approx-29 \mathrm{mV} / \mathrm{K}$ from $\approx 360$ down to $\approx 130 \mathrm{~K}$.

\section{Electrical Model}

At room temperature, the electrical frequency response of SiPMs was further investigated using a test device manufactured by STMicroelectronics. The latter device was a photodiode (PD) with a structure similar to that of a SPAD cell, an active area of $\approx 0.2 \mathrm{~mm}^{2}$, but no quenching resistor in series. In Fig. 6(a), the capacitance of the PD is shown as a function of reverse bias voltage at $100 \mathrm{~Hz}, 1 \mathrm{kHz}, 10 \mathrm{kHz}$ and $1 \mathrm{MHz}$. Furthermore, one can see that the device exhibits almost no dependence on the test frequency below $\approx 100 \mathrm{kHz}$ [Fig. 6(b)].

For silicon photodiodes and radiation detectors, the electrical response down to cryogenics temperatures is usually modeled using the so-called

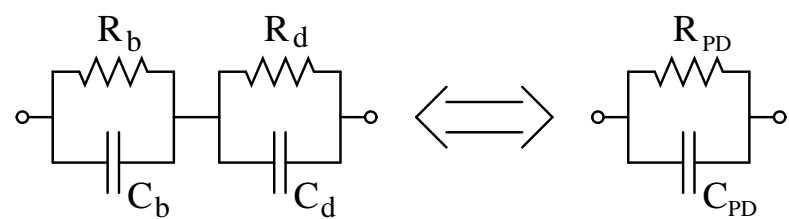

Fig. 7. SIJD model for silicon photodiodes and radiation detectors: $C_{\mathrm{d}}\left(C_{\mathrm{b}}\right)$ and $R_{\mathrm{d}}$ $\left(R_{\mathrm{b}}\right)$ are the capacitance and resistance of the depleted (field free) region, respectively; $C_{\mathrm{PD}}$ and $R_{\mathrm{PD}}$ are the overall capacitance and resistance of the photodiode, respectively. 


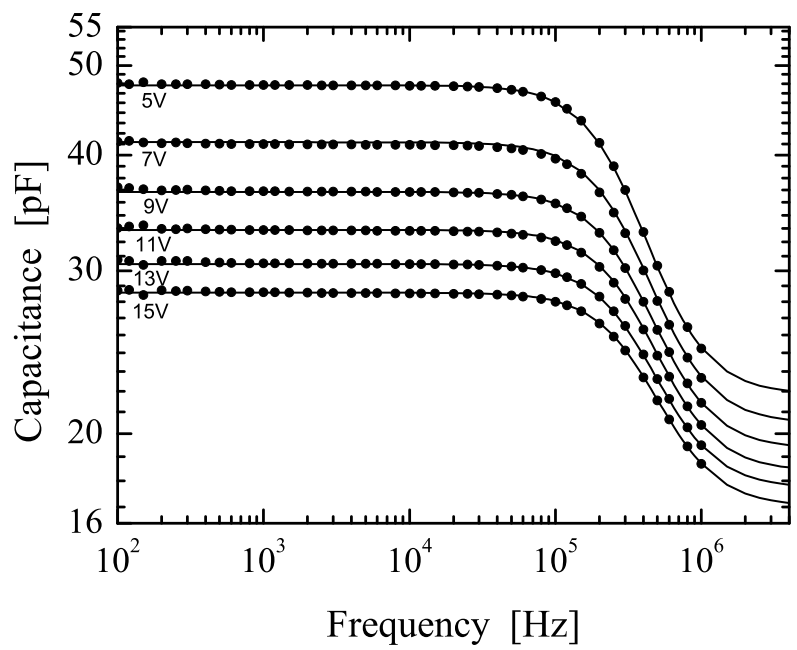

Fig. 8. Measured capacitance $(\bullet), C_{\mathrm{PD}}$, (in $\mathrm{pF}$ ) as a function of test frequency (in $\mathrm{Hz}$ ) with superimposed continuous lines obtained from the SIJD model [Eq. (3)].

small-signal ac impedance of junction diode (SIJD) operated under reverse bias (e.g., see Refs. ${ }^{6,7}$, Section 4.3 .4 of Ref. $^{8}$ and references therein). In the framework of the SIJD model, the device consists of the depleted and field

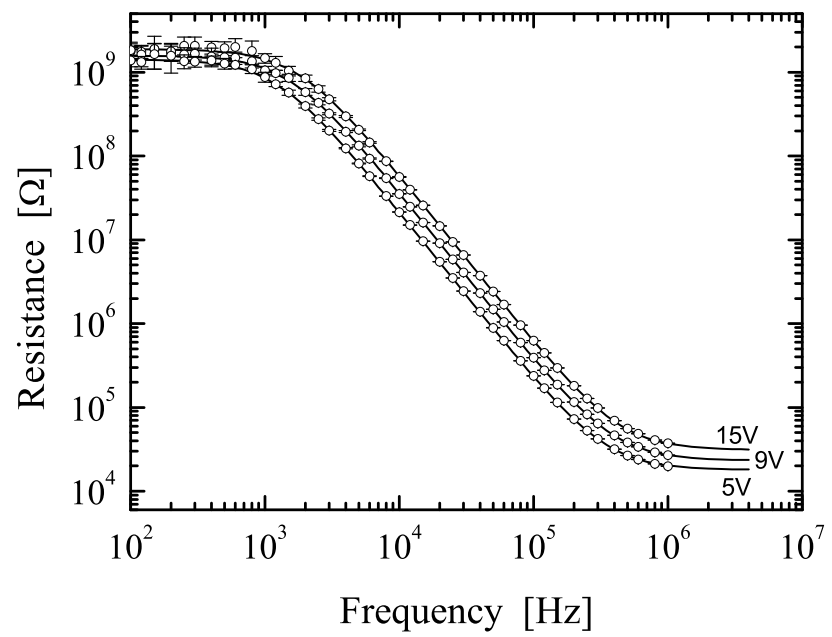

Fig. 9. Measured resistance (o), $R_{\mathrm{PD}}$, (in $\Omega$ ) as a function of test frequency (in $\mathrm{Hz}$ ) at 5 , 9 and $15 \mathrm{~V}$ with superimposed continuous lines obtained from the SIJD model [Eq. (2)]. 
8
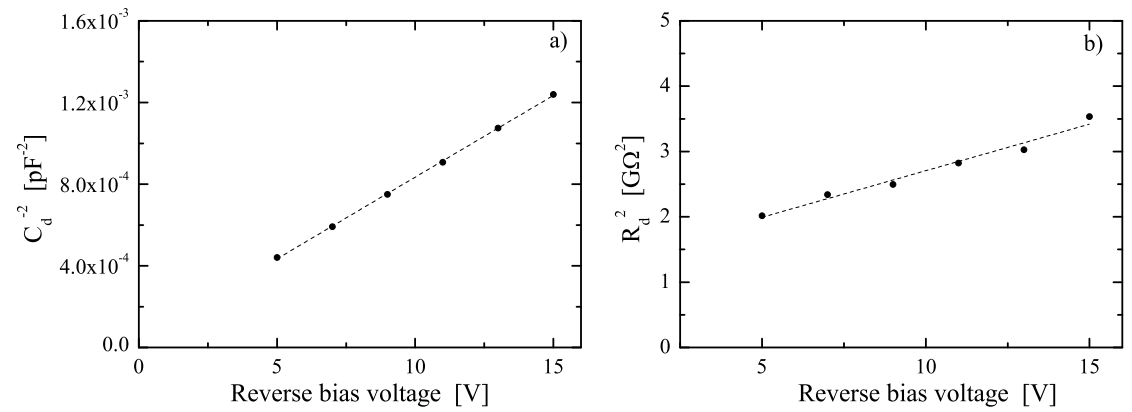

Fig. 10. (a) $1 / C_{\mathrm{d}}^{2}\left(\right.$ in $\mathrm{pF}^{-2}$ ) and (b) $R_{\mathrm{d}}^{2}\left(\right.$ in $\mathrm{G} \Omega^{2}$ ) from Table 1 as a function of applied reversed bias voltage in $\mathrm{V}$. The dashed lines are those from a linear fit to the data.

free regions connected in series. Each one of these regions, in turn, consists of a parallel-connected capacitor and resistor. In Fig. 7, the equivalent circuit of a photodiode is shown: $C_{\mathrm{d}}\left(C_{\mathrm{b}}\right)$ and $R_{\mathrm{d}}\left(R_{\mathrm{b}}\right)$ are the capacitance and resistance of the depleted (field free) region, respectively.

Labeling (Fig. 7) the overall ${ }^{\S}$ capacitance and resistance of a photodiode with $C_{\mathrm{PD}}$ and $R_{\mathrm{PD}}$, respectively, one can express the admittance of the device as:

$$
\begin{aligned}
Y_{\mathrm{PD}}(\omega) & =G_{\mathrm{PD}}(\omega)+j B_{\mathrm{PD}}(\omega) \\
& =\frac{1}{R_{\mathrm{PD}}}+j \omega C_{\mathrm{PD}}
\end{aligned}
$$

with $\omega=2 \pi f$, where $f$ is the test frequency. The conductance (i.e., the real part of the admittance) and susceptance (the imaginary part of the admittance) are respectively given by:

$$
\begin{aligned}
& G_{\mathrm{PD}}(\omega)=\frac{1}{R_{\mathrm{PD}}}=\frac{R_{\mathrm{d}}+R_{\mathrm{b}}+\omega^{2} R_{\mathrm{d}} R_{\mathrm{b}}\left(R_{\mathrm{d}} C_{\mathrm{d}}^{2}+R_{\mathrm{b}} C_{\mathrm{b}}^{2}\right)}{\left(R_{\mathrm{d}}+R_{\mathrm{b}}\right)^{2}+\omega^{2} R_{\mathrm{d}}^{2} R_{\mathrm{b}}^{2}\left(C_{\mathrm{b}}+C_{\mathrm{d}}\right)^{2}} \\
& B_{\mathrm{PD}}(\omega)=\omega C_{\mathrm{PD}}=\omega\left[\frac{\omega^{2} R_{\mathrm{d}}^{2} R_{\mathrm{b}}^{2} C_{\mathrm{d}} C_{\mathrm{b}}\left(C_{\mathrm{b}}+C_{\mathrm{d}}\right)+R_{\mathrm{d}}^{2} C_{\mathrm{d}}+R_{\mathrm{b}}^{2} C_{\mathrm{b}}}{\left(R_{\mathrm{d}}+R_{\mathrm{b}}\right)^{2}+\omega^{2} R_{\mathrm{d}}^{2} R_{\mathrm{b}}^{2}\left(C_{\mathrm{b}}+C_{\mathrm{d}}\right)^{2}}\right]
\end{aligned}
$$

[e.g., see Equations $(4.184,4.185)$ at page 455 of Ref. ${ }^{8}$.

It has to be remarked that the values of both $C_{\mathrm{PD}}$ and $R_{\mathrm{PD}}$ are those which can be determined, for instance, selecting the parallel equivalentcircuit mode for the DUT using the LCR meter. In the SIJD model, the values of $C_{\mathrm{d}}, R_{\mathrm{d}}, C_{\mathrm{b}}$ and $R_{\mathrm{b}}$ do not depend on the test frequency. In

${ }_{\S_{C} C_{\mathrm{PD}} \text { and } R_{\mathrm{PD}} \text { are the quantities }}$ directly measured selecting the parallel equivalentcircuit mode of the LCR and LCZ meters. 
Table 1. $C_{\mathrm{d}}, R_{\mathrm{d}}, C_{\mathrm{b}}$ and $R_{\mathrm{b}}$ obtained from a fit of the SIJD model for a photodiode to experimental data as a function of reverse bias voltage $\left(V_{\mathrm{r}}\right)$.

\begin{tabular}{r|cccc}
\hline$V_{\mathrm{r}}[\mathrm{V}]$ & $C_{\mathrm{d}}[\mathrm{pF}]$ & $R_{\mathrm{d}}[\mathrm{G} \Omega]$ & $C_{\mathrm{b}}[\mathrm{pF}]$ & $R_{\mathrm{b}}[\mathrm{k} \Omega]$ \\
\hline 5 & 47.6 & 1.42 & 41.1 & 5.2 \\
7 & 41.1 & 1.53 & 40.8 & 5.2 \\
9 & 36.5 & 1.58 & 40.8 & 5.2 \\
11 & 33.2 & 1.68 & 40.4 & 5.2 \\
13 & 30.5 & 1.74 & 40.9 & 5.3 \\
15 & 28.4 & 1.88 & 40.5 & 5.3 \\
\hline
\end{tabular}

addition, $C_{\mathrm{d}}, R_{\mathrm{d}}$ are expected to depend on the applied reverse bias voltage, because the depleted layer width increases with increasing $V_{\mathrm{r}}$.

$C_{\mathrm{d}}, R_{\mathrm{d}}, C_{\mathrm{b}}$ and $R_{\mathrm{b}}$ were determined (Table 1 ) as a function $V_{\mathrm{r}}$, by a fit to the measured quantities $C_{\mathrm{PD}}$ and $R_{\mathrm{PD}}$ (obtained using the LCR meter) using the corresponding expressions [e.g., see Eqs. $(2,3)$ ] obtained from the SIJD model for a photodiode. For instance, in Fig. 8 (Fig. 9) the experimental data and fitted curves are shown for the capacitance (resistance) measurements as a function of test frequency and applied reverse bias voltage. As expected ${ }^{6}$ (Table 1), the field free region is almost independent of $V_{\mathrm{r}}$, while both the capacitance and resistance of the depleted region exhibit a dependence on the reverse bias. In Fig. 10, $1 / C_{\mathrm{d}}^{2}$ [Fig. 10(a)] and $R_{\mathrm{d}}^{2}$ [Fig. 10(b)] values from Table 1 are shown as a function of applied reverse voltage. Although the junction photodiode cannot be considered as a onesided step junction ", the the dashed curves (Fig. 10) obtained from fits to the reported data - assuming a linear dependence of $1 / C_{\mathrm{d}}^{2}$ and $R_{\mathrm{d}}^{2}$ on $V_{\mathrm{r}}$ are well suited for reproducing the dependence on the reverse bias voltage.

\subsection{Electrical Model for SiPMs}

A SiPM device consists of a set of SPAD devices (4096 in the current SiPM under test) connected in parallel. The equivalent electrical circuit of the elemental cell (i.e., a SPAD cell) is shown in Fig. 11 and differs from that of a photodiode (discussed in Sect. 3 ) by a resistance $\left(R_{\mathrm{S}}\right)$ added in series. In Fig. $11, C_{\mathrm{d}}\left(C_{\mathrm{b}}\right)$ and $R_{\mathrm{d}}\left(R_{\mathrm{b}}\right)$ are the capacitance and resistance of the depleted (field free) region of the photodiode, respectively. In the present

TThe depletion layer characteristics as a function of reverse bias voltage can be found treated, for instance, in Chapter 6-2 of Ref. ${ }^{10}$ (see also Ref. ${ }^{9}$ ). 
10

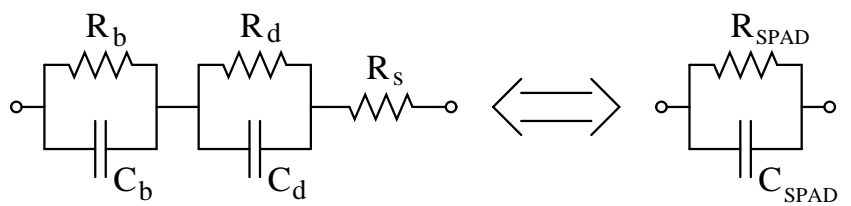

Fig. 11. SIJD model for a SPAD cell: $C_{\mathrm{d}}\left(C_{\mathrm{b}}\right)$ and $R_{\mathrm{d}}\left(R_{\mathrm{b}}\right)$ are, respectively, the capacitance and resistance of the depleted (field free) region with a series resistance $R_{\mathrm{s}}$; $C_{\text {spad }}$ and $R_{\text {spad }}$ are the overall capacitance and resistance of a SPAD cell, respectively.

technology of STMicroelectronics, $R_{\mathrm{s}}$ is typically about $(0.2-1.0) \mathrm{M} \Omega$.

The admittance $\left(Y_{\text {spad }}\right)$ of a SPAD cell is given by

$$
\begin{aligned}
Y_{\text {spad }}(\omega) & =G_{\text {spad }}(\omega)+j B_{\text {spad }}(\omega) \\
& =\frac{1}{R_{\text {spad }}}+j \omega C_{\text {spad }}
\end{aligned}
$$

with $C_{\text {spad }}$ and $R_{\text {spad }}$ respectively expressed in terms of $C_{\mathrm{d}}, C_{\mathrm{b}}, R_{\mathrm{d}}, R_{\mathrm{b}}$ and $R_{\mathrm{s}}$ as

$$
\begin{aligned}
& C_{\text {spad }}=\frac{B_{\text {spad }}(\omega)}{\omega} \\
& =\frac{\omega^{2} R_{\mathrm{d}}^{2} R_{\mathrm{b}}^{2} C_{\mathrm{d}} C_{\mathrm{b}}\left(C_{\mathrm{b}}+C_{\mathrm{d}}\right)+R_{\mathrm{d}}^{2} C_{\mathrm{d}}+R_{\mathrm{b}}^{2} C_{\mathrm{b}}}{D_{1}+D_{2}}, \\
& R_{\text {spad }}=\frac{1}{G_{\text {spad }}(\omega)} \\
& =\frac{D_{1}+D_{2}}{R_{\mathrm{b}}+\left(1+\omega^{2} C_{\mathrm{b}}^{2} R_{\mathrm{b}}^{2}\right)\left(R_{\mathrm{s}}+R_{\mathrm{d}}\right)+\omega^{2} R_{\mathrm{d}}^{2} C_{\mathrm{d}}^{2}\left(\omega^{2} C_{\mathrm{b}}^{2} R_{\mathrm{s}} R_{\mathrm{b}}^{2}+R_{\mathrm{s}}+R_{\mathrm{b}}\right)},(6) \\
& \mathrm{R}_{\mathrm{SPAD}}
\end{aligned}
$$

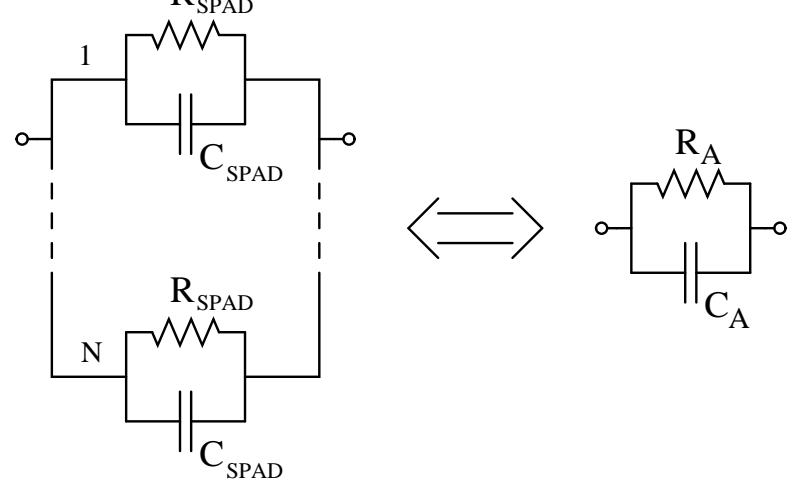

Fig. 12. SIJD model for a SiPM device consisting of N SPAD devices connected in parallel: $C_{\text {spad }}$ and $R_{\text {spad }}$ are, respectively, the capacitance and resistance of the equivalent circuit of a SPAD cell (Fig 11), $C_{\mathrm{A}}$ and $R_{\mathrm{A}}$ those of the SiPM device. 


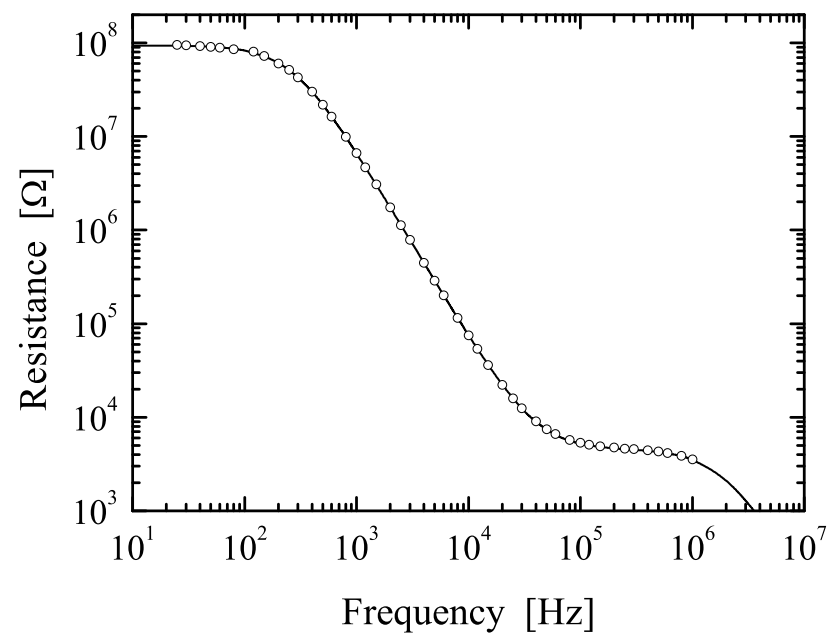

Fig. 13. Measured resistance (o), $R_{\mathrm{A}}$, (in $\Omega$ ) as a function of frequency (in $\mathrm{Hz}$ ) with superimposed the continuous line obtained from Eq. (8).

where $D_{1}$ and $D_{2}$ are

$$
\begin{aligned}
& D_{1}=\omega^{2} R_{\mathrm{b}}^{2} C_{\mathrm{b}}^{2}\left(R_{\mathrm{s}}+R_{\mathrm{d}}\right)^{2}+\omega^{2} R_{\mathrm{d}}^{2} C_{\mathrm{d}}^{2}\left(R_{\mathrm{s}}+R_{\mathrm{b}}\right)^{2} \\
& D_{2}=\left(R_{\mathrm{d}}+R_{\mathrm{b}}+R_{\mathrm{s}}\right)^{2}+\omega^{2} R_{\mathrm{d}}^{2} R_{\mathrm{b}}^{2} C_{\mathrm{d}} C_{\mathrm{b}}\left(2+\omega^{2} R_{\mathrm{s}}^{2} C_{\mathrm{d}} C_{\mathrm{b}}\right) .
\end{aligned}
$$

The equivalent electrical circuit* of a SiPM is shown in Fig. 12. Assuming that each SPAD cell has the same admittance, one finds that the overall admittance of an array is determined by:

$$
\begin{aligned}
Y_{\mathrm{A}}(\omega) & =N Y_{\text {spad }}(\omega) \\
& =G_{\mathrm{A}}(\omega)+j B_{\mathrm{A}}(\omega) \\
& =\frac{1}{R_{\mathrm{A}}}+j \omega C_{\mathrm{A}}
\end{aligned}
$$

with

$$
\begin{aligned}
G_{\mathrm{A}}(\omega) & =\frac{N}{R_{\text {spad }}} \\
B_{\mathrm{A}}(\omega) & =\omega N C_{\text {spad }},
\end{aligned}
$$

* It has to be remarked that one can also add a series resistance to $R_{\mathrm{A}}$. This was the case for another SiPM device with 3600 SPAD cells manufactured by STMicroelectronics; for such a device a small additional series resistance of about $1 \mathrm{k} \Omega$ was needed. 
Table 2. $C_{\mathrm{d}}, R_{\mathrm{d}}, C_{\mathrm{b}}, R_{\mathrm{b}}$ and $R_{\mathrm{s}}$ obtained from a fit of the SIJD model (adapted to a SiPM) to experimental data with the device operated at a reverse bias voltage of $5 \mathrm{~V}$.

\begin{tabular}{r|ccccc}
\hline$V_{\mathrm{r}}[\mathrm{V}]$ & $C_{\mathrm{d}}[\mathrm{pF}]$ & $R_{\mathrm{d}}[\mathrm{G} \Omega]$ & $C_{\mathrm{b}}[\mathrm{pF}]$ & $R_{\mathrm{b}}[\mathrm{M} \Omega]$ & $R_{\mathrm{S}}[\mathrm{k} \Omega]$ \\
\hline 5 & 0.262 & 385 & 0.056 & 12.5 & 195 \\
\hline
\end{tabular}

where $C_{\text {spad }}$ and $R_{\text {spad }}$ are obtained from Eqs. $(5,6)$, respectively; finally, $\mathrm{N}=64 \times 64=4096$ for the present device.

In Fig. 2 (13), the measured values of the capacitance (resistance) are shown as a function of test frequency up to $1 \mathrm{MHz}$, while the continuous line is that obtained using Eq. (9) [Eq. (8)]. These measurements were carried out at room temperature using the LCR meter with the SiPM device operated at a reverse bias voltage of $5 \mathrm{~V}$. Furthermore, in Table 2 the values of $C_{\mathrm{d}}, R_{\mathrm{d}}, C_{\mathrm{b}}, R_{\mathrm{b}}$ and $R_{\mathrm{s}}$ obtained from such a fit of the SIJD model (adapted to a SiPM) to experimental data are reported. It can be remarked that the value obtained for $R_{\mathrm{s}}$ is in agreement with one of those typically used in the current technology.

Finally, one can point out that the frequency dependence of the present SIJD model ${ }^{\|}$for SiPM and photodiode devices is well in agreement with measurements.

\section{Conclusions}

The electrical characteristics of SiPM devices were investigated as a function of applied reverse bias voltage $\left(V_{\mathrm{r}}\right)$ and temperature from 410 down to $100 \mathrm{~K}$. The capacitance response was also studied as a function of test frequency. One finds that the measured capacitance decreases i) with increasing the test frequency above $10 \mathrm{kHz}$ at $300 \mathrm{~K}$ and ii) with the decreasing of the temperature. Furthermore, the measurement of the leakage current allowed one to determine the value (and its temperature dependence) of the threshold voltage $\left(V_{\mathrm{th}}\right)$ above which a SiPM begins to operate in Geiger mode: $V_{\text {th }}$ decreases with lowering temperature at the rate of $\approx-29 \mathrm{mV} / \mathrm{K}$ from 300 down to $100 \mathrm{~K}$.

It was developed an electrical model to treat the frequency dependence of a SiPM device operated under reverse bias voltage. This model is based on the so-called small-signal ac impedance of junction diode (SIJD) used

${ }^{\|}$The reader can found modelizations of SiPM devices adapted for a time based readout ${ }^{11}$ or physical parameters measured at fixed frequencies (e.g., at $100 \mathrm{kHz}$ in Ref. ${ }^{5}$ and $1 \mathrm{MHz}$ in Ref. $\left.{ }^{12}\right)$. 
for radiation detectors and photodiodes. The model was found to be well suited to account for the SiPM dependencies of capacitance and resistance on frequency.

\section{References}

1. V.D. Kovaltchouk et al., Nucl. Instr. and Meth. in Phys. Res. A 538 (2005), 408-415.

2. M. Mazzillo et al., Nucl. Instr. and Meth. in Phys. Res. A 591 (2008), 367373.

3. F. Zappa et al., Sens. Actuat. A 140 (2007), 103-112.

4. M. Mazzillo et al., Sens. Actuat. A 138 (2007), 306-312.

5. G. Condorelli et al., Nucl. Instr. and Meth. in Phys. Res. A 654 (2011), 127-134.

6. C.H. Champness, J. of App. Phys. 62 (1987), 917.

7. C. Leroy and P.G. Rancoita, Particle Interaction and Displacement Damage in Silicon Devices operated in Radiation Environments, Rep. Prog. in Phys. 70 (2007), 403-625, doi: 10.1088/0034-4885/70/4/R01.

8. C. Leroy and P.G. Rancoita, Principles of Radiation Interaction in Matter and Detection - 3rd Edition - (2011), World Scientific, Singapore, ISBN-978981-4360-51-7.

9. R.B. Fair, J. Electrochem. Soc.: Solid State Sci., 118 (1971), 971.

10. Wolf, H.F. (1971). Semiconductors Wiley-Interscience, New York.

11. P. Jarron et al., IEEE Nuclear Science Symposium Conference Record (NSS/MIC) (2009), 1212-1219, doi: 10.1109/NSSMIC.2009.5402391.

12. F. Corsi et al., Nucl. Instr. and Meth. in Phys. Res. A 572 (2007), 416. 